\title{
Parametric Study on the Influence of Warping Deformation upon Natural Frequencies of Die Springs
}

\author{
Ying Hao and Junfeng Guan \\ School of Civil Engineering and Communication, North China University of Water Resources and Electric Power, \\ Zhengzhou 450045, China
}

Correspondence should be addressed to Ying Hao; yinghao_ncwu@163.com

Received 23 February 2016; Revised 17 May 2016; Accepted 22 May 2016

Academic Editor: Nuno M. Maia

Copyright (c) 2016 Y. Hao and J. Guan. This is an open access article distributed under the Creative Commons Attribution License, which permits unrestricted use, distribution, and reproduction in any medium, provided the original work is properly cited.

\begin{abstract}
The free vibrational characteristics of die springs are examined by Riccati transfer matrix method in this study. The warping deformation of spring's cross section, as a new design factor, is incorporated into the differential equation of motion. Numerical simulations show that the warping deformation is a significant role of the behavior of natural frequencies of die springs and should be considered carefully. Approximately $40 \%$ of the errors may occur if warping is neglected. The change laws of warping effect with the parameter variations of springs are also explored, including the height-to-width ratio of the cross section, the cylinder diameter, the helix pitch angle, and the number of coils. The warping effect exhibits the most remarkable changes with the variation in the height-to-width ratio of the cross section. However, this effect is not fairly sensitive to the changes in other parameters, and it is particularly significant when the cross section is relatively narrow regardless of the changes in other parameters. This study evidently answers the key scientific question: "under what working condition should the warping effect be considered or ignored?" The analysis results can be used to guide spring designers in engineering.
\end{abstract}

\section{Introduction}

Die spring usually refers to a cylindrical helical metal spring with rectangular cross section (see Figure 3). Due to the advantages of high and almost constant rigidity, large energy storage, nearly linear characteristics, and small size, it is suitable to be used for limited space. Therefore, it has been widely applied in the precision machinery and automotive fields.

The studies on the free vibration of cylindrical helical springs have been carried by many scholars [1-4]; however the shapes of wire cross sections in those researches are mostly circular. The Saint-Venant torsion problem of nonhomogeneous bars with solid and hollow cross sections was treated by Ecsedi [5]. The shear modulus of the nonhomogeneous bar was a function which matched Prandtl's stress function of the cylindrical bar assuming that it is made of the homogeneous material. Only a few studies on buckling behavior [6] and vibration characteristics [710] of springs with rectangular cross sections have been conducted; however, warping deformation has been ignored in these analyses. Recently, Yu and Hao [11] demonstrated the first free vibration analysis of cylindrical helical springs and warping effect by employing theoretical analysis and Muller root search method. The effects of all parameters, such as the height-to-width ratio of the cross section, the cylinder diameter, the helix pitch angle, and the number of coils upon natural frequency, had been discussed; only the change laws of warping effect with the parameter variations were not systematically examined and that method applied was complicated by low efficiency [11].

Based on the naturally curved and twisted beam theory $[12,13]$, a new series of differential equations of motion for die springs with warping deformation is derived in this study. This series is composed of 14 coupled first-order partial differential equations. Natural frequencies are solved 
by improving Riccati transfer matrix method, and the validity of the proposed model is verified comparing ANSYS results and the published literature.

The analysis results in this paper show that if the cross section of a spring wire is relatively long and narrow, it will induce $43.06 \%$ error to the fundamental frequency with the warping being ignored. On the contrary, the error can be reduced to $0.37 \%$ if the warping effect was considered. Numerical results show that the warping effect exhibits the most remarkable changes with the variation of the height-towidth ratio of the cross section. Therefore, the suggestions can be given as follows: when the aspect ratio of the rectangular cross section is $a: b=1: 0.6$, warping deformation should be considered in die spring's dynamic analysis; when the aspect ratio is $a: b=1: 0.4$, the influence of warping must be considered. However, the effects are not fairly sensitive to the changes of other parameters; in particular when the cross section is relatively narrow, the changes in other parameters can be ignored.

\section{Differential Equations of Motion for Die Springs with Warping Effect}

2.1. The Geometric Compatibility and Constitutive Equations. Consider a naturally curved and twisted beam in space in Figure 1. The tangential, normal, and binormal unit vectors of the beam axis are expressed as $\mathbf{t}, \mathbf{n}$, and $\mathbf{b}$, respectively. For a smooth curve, the Frenet-Serret formulae are

$$
\begin{aligned}
\mathbf{t}^{\prime} & =k_{1} \mathbf{n}, \\
\mathbf{n}^{\prime} & =-k_{1} \mathbf{t}+k_{2} \mathbf{b}, \\
\mathbf{b}^{\prime} & =-k_{2} \mathbf{n},
\end{aligned}
$$

where ()$^{\prime}=d / d s, s, k_{1}, k_{2}$ are arc coordinate, curvature, and torsion of the curve, respectively. In order to take into account the initial twist of the cross section, a new Cartesian reference frame $\left(x_{1}, \xi, \eta\right)$ is introduced, in which the $x_{1}$-axis is in the direction of $\mathbf{t}$ and the angle between the $\xi$-axis and $\mathbf{n}$-axis is $\theta(s)$. If the unit vectors of $O \xi$ and $O \eta$ are represented by $\mathbf{i}_{\xi}$ and $\mathbf{i}_{\eta}$, then

$$
\begin{aligned}
& \mathbf{i}_{\xi}=\mathbf{n} \cos \theta+\mathbf{b} \sin \theta, \\
& \mathbf{i}_{\eta}=-\mathbf{n} \sin \theta+\mathbf{b} \cos \theta .
\end{aligned}
$$

Simultaneously solving (1) and (2), we have

$$
\left\{\begin{array}{c}
\mathbf{t}^{\prime} \\
\mathbf{i}_{\xi}^{\prime} \\
\mathbf{i}_{\eta}^{\prime}
\end{array}\right\}=\left[\begin{array}{ccc}
0 & k_{\eta} & -k_{\xi} \\
-k_{\eta} & 0 & k_{s} \\
k_{\xi} & -k_{s} & 0
\end{array}\right]\left\{\begin{array}{c}
\mathbf{t} \\
\mathbf{i}_{\xi} \\
\mathbf{i}_{\eta}
\end{array}\right\},
$$

where $k_{\xi}=k_{1} \sin \theta, k_{\eta}=k_{1} \cos \theta, k_{s}=k_{2}+\theta^{\prime}$.

Assuming that the cross section has infinite rigidity in its own plane but is free to warp out of plane and the deformation

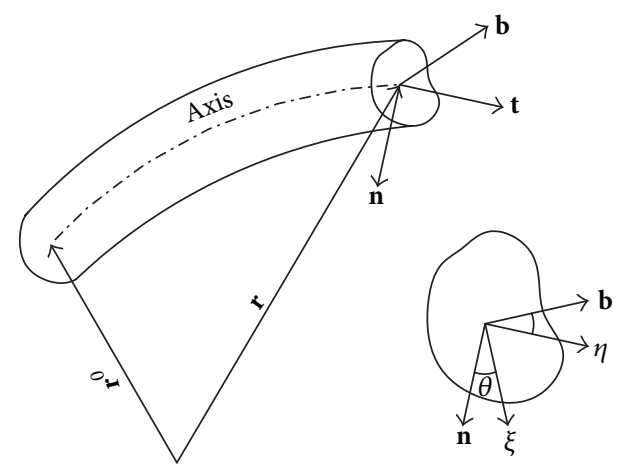

FIGURE 1: Geometry of a naturally curved and twisted beam.

of the beam is caused by the extension, bending and torsion can be expressed as $[13,14]$

$$
\begin{aligned}
\mathbf{u}(s, \xi, \eta, t)= & W(s, \xi, \eta, t) \mathbf{t}+U(s, \xi, \eta, t) \mathbf{i}_{\xi} \\
& +V(s, \xi, \eta, t) \mathbf{i}_{\eta},
\end{aligned}
$$

where

$$
\begin{aligned}
U(s, \xi, \eta, t)= & u_{\xi}(s, t)-\eta \varphi_{s}(s, t) \\
V(s, \xi, \eta, t)= & u_{\eta}(s, t)+\xi \varphi_{s}(s, t) \\
W(s, \xi, \eta, t)= & u_{s}(s, t)+\eta \varphi_{\xi}(s, t)-\xi \varphi_{\eta}(s, t) \\
& +\alpha(s, t) \varphi(\xi, \eta)
\end{aligned}
$$

where $u_{s}(s), u_{\xi}(s)$, and $u_{\eta}(s)$ are the three translations, $\varphi_{s}(s)$, $\varphi_{\xi}(s)$, and $\varphi_{\eta}(s)$ are the three rotations, respectively, and $\alpha(s)$ is a generalized warping coordinate. And $\varphi(\xi, \eta)$ is the warping function of Saint-Venant's torsion of a cylindrical shaft which has the same cross section as the beam under consideration.

Let $e_{s s}, e_{s \xi}, e_{s \eta}$ be the axial and shear strain components, respectively,

$$
\begin{aligned}
\sqrt{g} e_{s s}= & \varepsilon_{s}+\eta \omega_{\xi}-\xi \omega_{\eta}+\sqrt{g} \varphi \alpha^{\prime} \\
& +k_{s}\left[\left(\frac{\partial \varphi}{\partial \xi}\right) \eta-\left(\frac{\partial \varphi}{\partial \eta}\right) \xi\right] \alpha, \\
2 \sqrt{g} e_{s \xi}= & G_{\xi} \varepsilon_{\xi}-\varphi_{\eta}-\eta \omega_{s}+\left[\sqrt{g}\left(\frac{\partial \varphi}{\partial \xi}\right)+k_{\eta} \varphi\right] \alpha, \\
2 \sqrt{g} e_{s \eta}= & G_{\eta} \varepsilon_{\eta}+\varphi_{\xi}+\xi \omega_{s}+\left[\sqrt{g}\left(\frac{\partial \varphi}{\partial \eta}\right)+k_{\xi} \varphi\right] \alpha .
\end{aligned}
$$

In which $G_{\xi}, G_{\eta}$ are shear correction factors and $g=\left(1-\xi k_{\eta}+\right.$ $\left.\eta k_{\xi}\right)^{2}$. Based on the assumption that the initial curvature of the beam is gentle, it can be seen that $\sqrt{g} \approx 1$. Let

$$
\begin{aligned}
& \varepsilon_{s}=u_{s}^{\prime}-k_{\eta} u_{\xi}+k_{\xi} u_{\eta}, \\
& \varepsilon_{\xi}=u_{\xi}^{\prime}-k_{s} u_{\eta}+k_{\eta} u_{s}, \\
& \varepsilon_{\eta}=u_{\eta}^{\prime}-k_{\xi} u_{s}+k_{s} u_{\xi},
\end{aligned}
$$




$$
\begin{aligned}
& \omega_{s}=\varphi_{s}^{\prime}-k_{\eta} \varphi_{\xi}+k_{\xi} \varphi_{\eta}, \\
& \omega_{\xi}=\varphi_{\xi}^{\prime}-k_{s} \varphi_{\eta}+k_{\eta} \varphi_{s}, \\
& \omega_{\eta}=\varphi_{\eta}^{\prime}-k_{\xi} \varphi_{s}+k_{s} \varphi_{\xi} .
\end{aligned}
$$

For the case of isotropic beam under consideration, the stress components can be expressed as follows:

$$
\begin{aligned}
\sigma_{s} & =E e_{s s}, \\
\tau_{s \xi} & =2 G e_{s \xi}, \\
\tau_{s \eta} & =2 G e_{s \eta},
\end{aligned}
$$

where $E, G$ are elastic modulus and shear modulus of the material, respectively.

The resultant forces $\mathbf{Q}(s, t)=\left\{Q_{s}^{\mathrm{ex}}(s, t), Q_{\xi}^{\mathrm{ex}}(s, t), Q_{\eta}^{\mathrm{ex}}(s\right.$, $t)\}$ and moments $\mathbf{M}(s, t)=\left\{M_{s}^{\mathrm{ex}}(s, t), M_{\xi}^{\mathrm{ex}}(s, t), M_{\eta}^{\mathrm{ex}}(s, t)\right\}$ on the cross section are shown as follows:

$$
\begin{aligned}
Q_{s} & =\iint \sigma_{s} d \xi d \eta, \\
Q_{\xi} & =\iint \tau_{s \xi} d \xi d \eta, \\
Q_{\eta} & =\iint \tau_{s \eta} d \xi d \eta, \\
M_{s} & =\iint\left(\tau_{s \eta} \xi-\tau_{s \xi} \eta\right) d \xi d \eta, \\
M_{\xi} & =\iint \sigma_{s} \eta d \xi d \eta, \\
M_{\eta} & =-\iint \sigma_{s} \xi d \xi d \eta, \\
T & =\iint \sigma_{s} \varphi d \xi d \eta,
\end{aligned}
$$

where $Q_{s}$ is the axial force, $Q_{\xi}$ and $Q_{\eta}$ are the shear forces, $M_{s}$ is the twisting moment, $M_{\xi}$ and $M_{\eta}$ are the bending moments, and $T$ is the generalized warping moment.

2.2. The Equations of Motion. The external force and moments per unit length of the beam axis can be expressed as

$$
\begin{aligned}
\mathbf{q}^{\mathrm{ex}}(s, t) & =\left\{q_{s}^{\mathrm{ex}}(s, t), q_{\xi}^{\mathrm{ex}}(s, t), q_{\eta}^{\mathrm{ex}}(s, t)\right\}, \\
\mathbf{m}^{\mathrm{ex}}(s, t) & =\left\{m_{s}^{\mathrm{ex}}(s, t), m_{\xi}^{\mathrm{ex}}(s, t), m_{\eta}^{\mathrm{ex}}(s, t)\right\} .
\end{aligned}
$$

Meanwhile, $\mathbf{q}^{\text {in }}(s, t)=\left\{q_{s}^{\text {in }}(s, t), q_{\xi}^{\text {in }}(s, t), q_{\eta}^{\text {in }}(s, t)\right\}, \mathbf{m}^{\text {in }}(s, t)=$ $\left\{m_{s}^{\text {in }}(s, t), m_{\xi}^{\text {in }}(s, t), m_{\eta}^{\text {in }}(s, t)\right\}$ denote the inertial force and the inertial moment per unit length of the rod axis. Since the displacements and the rotations are assumed to be infinitesimal, the equations of motion can be written as

$$
\begin{aligned}
& \frac{\partial \mathbf{Q}(s, t)}{\partial s}+\mathbf{q}^{\mathrm{ex}}(s, t)=\mathbf{q}^{\mathrm{in}}(s, t) ; \\
& \frac{\partial \mathbf{M}(s, t)}{\partial s}+\mathbf{t} \times \mathbf{Q}(s, t)+\mathbf{m}^{\mathrm{ex}}(s, t)=\mathbf{m}^{\mathrm{in}}(s, t) ; \\
& \frac{\partial T(s, t)}{\partial s}=\left(\iint \sigma_{s} \varphi d \xi d \eta\right)^{\prime},
\end{aligned}
$$

where

$$
\begin{aligned}
q_{s}^{\text {in }}(s, t)= & \iint_{A} \rho \frac{\partial^{2} u_{s}(s, t)}{\partial t^{2}} d \xi d \eta ; \\
q_{\xi}^{\text {in }}(s, t)= & \iint_{A} \rho \frac{\partial^{2} u_{\xi}(s, t)}{\partial t^{2}} d \xi d \eta ; \\
q_{\eta}^{\text {in }}(s, t)= & \iint_{A} \rho \frac{\partial^{2} u_{\eta}(s, t)}{\partial t^{2}} d \xi d \eta ; \\
m_{s}^{\text {in }}(s, t)= & \iint_{A} \rho \xi \frac{\partial^{2} u_{\eta}(s, t)}{\partial t^{2}} d \xi d \eta \\
& -\iint_{A} \rho \eta \frac{\partial^{2} u_{\xi}(s, t)}{\partial t^{2}} d \xi d \eta ; \\
m_{\xi}^{\text {in }}(s, t)= & \iint_{A} \rho \eta \frac{\partial^{2} u_{s}(s, t)}{\partial t^{2}} d \xi d \eta ; \\
m_{\eta}^{\text {in }}(s, t)= & -\iint_{A} \rho \xi \frac{\partial^{2} u_{s}(s, t)}{\partial t^{2}} d \xi d \eta .
\end{aligned}
$$

2.3. Differential Equations of Motion for Die Springs with Warping Effect. The geometrical properties of the die springs are given in Figure 2. The parametric relationships of a cylindrical helix can be denoted as

$$
\begin{aligned}
h & =R \tan \bar{\alpha}, \\
c & =\sqrt{R^{2}+h^{2}}, \\
k_{s} & =\frac{h}{c^{2}}, \\
k_{\eta} & =\frac{R}{c^{2}}, \\
d s & =c d \beta,
\end{aligned}
$$

where $\bar{\alpha}$ is the pitch angle, $R$ is the radius of helix, $h$ is the step for unit angle of the helix, and $k_{\eta}$ and $k_{s}$ are the curvature and torsion of the helix axis, respectively. $d \beta=d s / c$ is the infinitesimal angular element. 


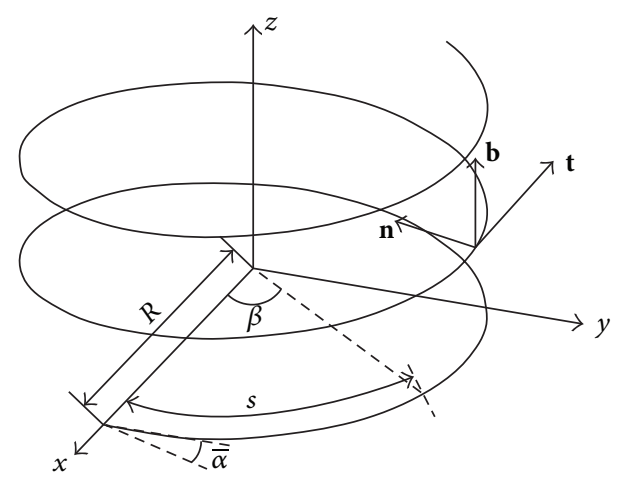

FIGURE 2: Geometry of a typical cylindrical helical spring.

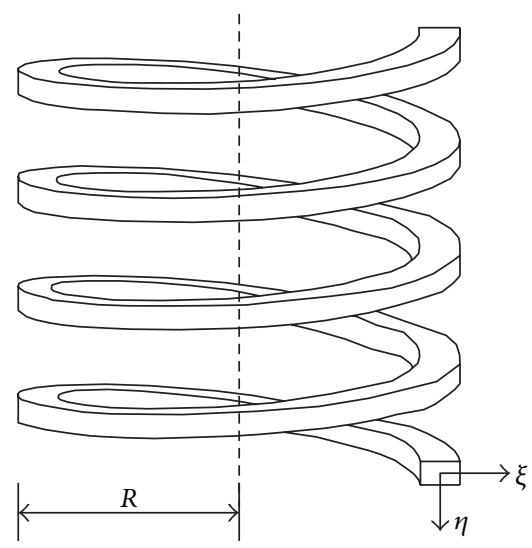

FIgURE 3: Cylindrical helical spring with rectangular cross section.

The differential equations of die spring can be simplified as follows by simultaneously solving (5)-(11):

$\frac{d u_{s}(\beta)}{d \beta}=\cos \bar{\alpha} u_{\xi}(\beta)-\frac{\lambda_{1} \sin \bar{\alpha}}{A} \alpha(\beta)+\frac{c}{E A} Q_{s}(\beta)$,

$\frac{d u_{\xi}(\beta)}{d \beta}=-\cos \bar{\alpha} u_{s}(\beta)+\sin \bar{\alpha} u_{\eta}(\beta)+c \varphi_{\eta}(\beta)$

$+\frac{c}{G G_{\xi} A} Q_{\xi}(\beta)$

$\frac{d u_{\eta}(\beta)}{d \beta}=-\sin \bar{\alpha} u_{\xi}(\beta)-c \varphi_{\xi}(\beta)+\frac{c}{G G_{\eta} A} Q_{\eta}(\beta)$,

$\frac{d \varphi_{s}(\beta)}{d \beta}=\cos \bar{\alpha} \varphi_{\xi}(\beta)-\frac{c\left(\lambda_{3}-\lambda_{2}\right)}{I_{2}+I_{3}} \alpha(\beta)$

$+\frac{c}{G\left(I_{2}+I_{3}\right)} M_{s}(\beta)$,

$\frac{d \varphi_{\xi}(\beta)}{d \beta}=-\cos \bar{\alpha} \varphi_{s}(\beta)+\sin \bar{\alpha} \varphi_{\eta}(\beta)+\frac{c}{E I_{2}} M_{\xi}(\beta)$,

$\frac{d \varphi_{\eta}(\beta)}{d \beta}=-\sin \bar{\alpha} \varphi_{\xi}(\beta)-\frac{c}{E I_{3}} M_{\eta}(\beta)$ $\frac{d \alpha(\beta)}{d \beta}=\frac{c}{E \lambda_{4}} T(\beta)$,

$\frac{d Q_{s}(\beta)}{d \beta}=\cos \bar{\alpha} Q_{\xi}(\beta)-c \rho A \omega^{2} u_{s}(\beta)$,

$\frac{d Q_{\xi}(\beta)}{d \beta}=-\cos \bar{\alpha} Q_{s}(\beta)+\sin \bar{\alpha} Q_{\eta}(\beta)$

$-c \rho A \omega^{2} u_{\xi}(\beta)$,

$\frac{d Q_{\eta}(\beta)}{d \beta}=-\sin \bar{\alpha} Q_{\xi}(\beta)-c \rho A \omega^{2} u_{\eta}(\beta)$,

$\frac{d M_{s}(\beta)}{d \beta}=\cos \bar{\alpha} M_{\xi}(\beta)-c \rho\left(I_{2}+I_{3}\right) \omega^{2} \varphi_{s}(\beta)$,

$\frac{d M_{\xi}(\beta)}{d \beta}=-\cos \bar{\alpha} M_{s}(\beta)+\sin \bar{\alpha} M_{\eta}(\beta)+c Q_{\eta}(\beta)$

$-c \rho I_{2} \omega^{2} \varphi_{\xi}(\beta)$,

$\frac{d M_{\eta}(\beta)}{d \beta}=-\sin \bar{\alpha} M_{\xi}(\beta)-c Q_{\xi}(\beta)-c \rho I_{3} \omega^{2} \varphi_{\eta}(\beta)$,

$\frac{d T(\beta)}{d \beta}=\frac{\lambda_{1} \sin \bar{\alpha}}{A} Q_{s}(\beta)+\frac{c\left(\lambda_{2}-\lambda_{3}\right)}{I_{2}+I_{3}} M_{s}(\beta)$

$+c\left[G\left(\lambda_{6}+\lambda_{7}\right)+k_{\eta}^{2} G \lambda_{4}+k_{s}^{2} E \lambda_{5}-\rho \lambda_{4} \omega^{2}\right.$

$\left.-\frac{k_{s}^{2} E \lambda_{1}^{2}}{A}-\frac{G\left(\lambda_{2}-\lambda_{3}\right)^{2}}{I_{2}+I_{3}}\right] \alpha(\beta)$

where

$$
\begin{aligned}
A & =\iint_{A} d \xi d \eta \\
I_{2} & =\iint_{A} \eta^{2} d \xi d \eta \\
I_{3} & =\iint_{A} \xi^{2} d \xi d \eta \\
\lambda_{1} & =\iint_{A}\left[\left(\frac{\partial \varphi}{\partial \xi}\right) \eta-\left(\frac{\partial \varphi}{\partial \eta}\right) \xi\right] d \xi d \eta, \\
\lambda_{2} & =\iint_{A} \xi \frac{\partial \varphi}{\partial \eta} d \xi d \eta, \\
\lambda_{3} & =\iint_{A} \eta \frac{\partial \varphi}{\partial \xi} d \xi d \eta, \\
\lambda_{4} & =\iint_{A} \varphi^{2} d \xi d \eta, \\
\lambda_{5} & =\iint_{A}\left[\left(\frac{\partial \varphi}{\partial \xi}\right) \eta-\left(\frac{\partial \varphi}{\partial \eta} \xi\right)\right]^{2} d \xi d \eta
\end{aligned}
$$




$$
\begin{aligned}
& \lambda_{6}=\iint_{A}\left(\frac{\partial \varphi}{\partial \xi}\right)^{2} d \xi d \eta, \\
& \lambda_{7}=\iint_{A}\left(\frac{\partial \varphi}{\partial \eta}\right)^{2} d \xi d \eta .
\end{aligned}
$$

\section{Calculation of Element Transfer Matrix}

Equations (14) is written in matrix form

$$
\frac{d}{d \beta} \mathbf{S}(\beta)=\mathbf{D}(\beta, \omega) \mathbf{S}(\beta),
$$

where

$$
\mathbf{D}(\beta, \omega) \quad \text { and } \quad \mathbf{S}(\beta)
$$

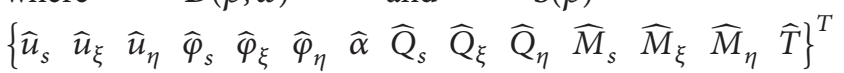
signify the differential matrix and the state vector, respectively. Dividing the die spring into small elements and on any element $\left[\beta_{i}, \beta_{i+1}\right]$, there is

$$
\mathbf{S}_{i+1}(\beta)=e^{\int_{\beta_{i}}^{\beta_{i+1}} \mathbf{D}(\beta, \omega) d \beta} \mathbf{S}_{i}(\beta)=\mathbf{F}_{i}(\omega) \mathbf{S}_{i}(\beta) .
$$

The equations become a "stiff-conditioning" system of equations because of the increase by two degrees of freedom containing the generalized warping coordinate and the generalized warping moment. Therefore, using the method of [14] to calculate the element transfer matrix $\mathbf{F}_{i}(\omega)$ is no longer applicable. Therefore, Scaling-Squaring method and Padé approximation formula are used to calculate the index matrix $\mathbf{F}_{i}(\omega)$ in this study.

For any matrix $\mathbf{A}$ and any positive integer $m$, one has

$$
e^{\mathbf{A}}=\left(e^{\mathbf{A} / m}\right)^{m} .
$$

Scaling-Squaring method can be used to calculate $e^{\mathbf{A}}$ into $e^{\mathbf{A} / m}$. And index matrix $e^{\mathbf{A} / m}$ can be calculated by Padé approximation formula. The approximate expression is

$$
e^{\mathbf{B}}=D_{p q}^{-1}(\mathbf{B}) N_{p q}(\mathbf{B})
$$

where

$$
\begin{aligned}
& D_{p q}(\mathbf{B})=\sum_{j=0}^{q} \frac{(p+q-j) ! q !}{(p+q) ! j !(q-j) !}(-\mathbf{B})^{j}, \\
& N_{p q}(\mathbf{B})=\sum_{j=0}^{p} \frac{(p+q-j) ! p !}{(p+q) ! j !(p-j) !} \mathbf{B}^{j} .
\end{aligned}
$$

\section{Improved Riccati Transfer Matrix Method}

After getting the element transfer matrix by Scaling-Squaring method and Padé approximation formula, the state vector of the motion differential equations (14) is decomposed into two states which are composed of seven elements:

$$
\begin{aligned}
& \mathbf{S}(\beta)
\end{aligned}
$$

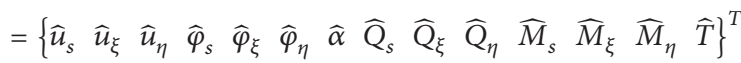

$$
\begin{aligned}
& =\left\{\mathbf{f}_{7 \times 1}, \mathbf{e}_{7 \times 1}\right\} \text {. }
\end{aligned}
$$

TABLE 1: Viability and effectiveness of the proposed model.

\begin{tabular}{lccccc}
\hline Mode number $(\mathrm{Hz})$ & 1 & 2 & 3 & 4 & 5 \\
\hline FEM [11] & 314.1 & 337.2 & 368.2 & 376.0 & 609.0 \\
Reference [11] & 313.2 & 336.8 & 366.7 & 375.5 & 607.9 \\
Present study & 314.0 & 338.3 & 368.9 & 376.8 & 609.2 \\
\hline
\end{tabular}

In formula (21), the vectors $\mathbf{f}$ and $\mathbf{e}$ should satisfy the boundary conditions of the initial cross section spring $(\beta=0)$. 16]

Then the unit transfer relationship can be rewritten as $[15$,

$$
\left\{\begin{array}{l}
\mathbf{f} \\
\mathbf{e}
\end{array}\right\}_{i+1}=\left[\begin{array}{ll}
\mathbf{F}_{11}(\omega) & \mathbf{F}_{12}(\omega) \\
\mathbf{F}_{21}(\omega) & \mathbf{F}_{22}(\omega)
\end{array}\right]_{i}\left\{\begin{array}{l}
\mathbf{f} \\
\mathbf{e}
\end{array}\right\}_{i}
$$

Substituting Riccati transformation $\mathbf{f}_{i}=\mathbf{S}_{i} \mathbf{e}_{i}$ into the above formula, one has

$$
\begin{aligned}
\mathbf{f}_{i+1} & \\
\quad= & {\left[\mathbf{F}_{11}(\omega) \mathbf{S}+\mathbf{F}_{12}(\omega)\right]_{i}\left[\mathbf{F}_{21}(\omega) \mathbf{S}+\mathbf{F}_{22}(\omega)\right]_{i}^{-1} \mathbf{e}_{i+1}, } \\
\mathbf{e}_{i}= & {\left[\mathbf{F}_{21}(\omega) \mathbf{S}+\mathbf{F}_{22}(\omega)\right]_{i}^{-1} \mathbf{e}_{i+1} . }
\end{aligned}
$$

The recursive relation of Riccati transformation is obtained:

$$
\mathbf{S}_{i+1}=\left[\mathbf{F}_{11}(\omega) \mathbf{S}+\mathbf{F}_{12}(\omega)\right]_{i}\left[\mathbf{F}_{21}(\omega) \mathbf{S}+\mathbf{F}_{22}(\omega)\right]_{i}^{-1} .
$$

According to (24), different frequency equation can be gotten by different types of boundary conditions of spring end section. For example, when the springs are at both ends with the same boundary conditions, the frequency equation is

$$
\left|\mathbf{S}_{N+1}\right| \prod_{i=1}^{N} \operatorname{sign}\left|\mathbf{F}_{21}(\omega) \mathbf{S}+\mathbf{F}_{22}(\omega)\right|_{i}=0 .
$$

According to the above method, the natural frequency and vibration mode of the die spring with fixed-fixed ends can be obtained.

\section{Applications}

Several numerical results are given in this section.

Example 1. Firstly, as shown in Table 1, a spring with an elliptic cross section and clamped-clamped ends is considered to verify the viability and effectiveness of the proposed model. The geometry properties are as follows: $R=5 \mathrm{~mm}, n=7.6$, $\bar{\alpha}=5^{\circ}$, and the semiaxes $a=0.3 \mathrm{~mm}$ in the $\xi$-direction and $b=0.5 \mathrm{~mm}$ in the $\eta$-direction. The computation time for the free vibration frequencies calculated by the Riccati method is 43 seconds, whereas the time calculated by the Muller root search method is 112 seconds [11].

Example 2. Springs with rectangular cross section and clamped-clamped ends are shown in Examples 2-6 with the following material and geometry properties: $\rho=7900 \mathrm{~kg} / \mathrm{m}^{3}$, $E=2.06 \times 10^{11} \mathrm{~N} / \mathrm{m}^{2}, \mu=0.3$, the cylinder diameter $R$, the helix pitch angle $\bar{\alpha}$, the number of active turns $n$, and the rectangular cross section of the semiaxes $a$ in the $\xi$-direction 
TABLE 2: Comparison of the first five frequencies of the spring.

\begin{tabular}{|c|c|c|c|c|c|}
\hline \multirow{2}{*}{ Mode number $(\mathrm{Hz})$} & \multirow{2}{*}{ FEM } & \multicolumn{4}{|c|}{ Present study (PS) } \\
\hline & & Warping ignored & Errors (\%) & Warping included & Errors (\%) \\
\hline 1 & 521.34 & 745.84 & 43.06 & 523.25 & 0.37 \\
\hline 2 & 899.54 & 1009.30 & 12.20 & 897.71 & 0.20 \\
\hline 3 & 915.97 & 1044.17 & 13.99 & 914.36 & 0.17 \\
\hline 4 & 970.21 & 1122.22 & 15.67 & 973.88 & 0.38 \\
\hline 5 & 1129.5 & 1338.65 & 18.52 & 1118.41 & 0.98 \\
\hline
\end{tabular}

TABLE 3: Change law of the warping effect on natural frequencies with respect to the aspect ratio $(a / b)$ of the rectangular cross section.

\begin{tabular}{|c|c|c|c|c|c|c|}
\hline \multicolumn{2}{|c|}{ Mode number $(\mathrm{Hz})$} & 1 & 2 & 3 & 4 & 5 \\
\hline \multirow{5}{*}{$\begin{array}{l}2 a=1 \mathrm{~mm} \\
2 b=0.4 \mathrm{~mm}\end{array}$} & FEM & 521.34 & 899.54 & 915.97 & 970.21 & 1129.5 \\
\hline & Warping ignored & 745.84 & 1009.3 & 1044.1 & 1122.2 & 1338.6 \\
\hline & Error (\%) & 43.06 & 12.20 & 13.99 & 15.67 & 18.52 \\
\hline & Warping included & 523.25 & 897.71 & 914.36 & 973.88 & 1118.4 \\
\hline & Error (\%) & 0.37 & 0.20 & 0.17 & 0.38 & 0.98 \\
\hline \multirow{5}{*}{$\begin{array}{l}2 a=1 \mathrm{~mm} \\
2 b=0.6 \mathrm{~mm}\end{array}$} & FEM & 718.70 & 1136.7 & 1170.0 & 1209.3 & 1339.7 \\
\hline & Warping ignored & 817.31 & 1128.6 & 1207.1 & 1251.5 & 1511.9 \\
\hline & Error (\%) & 13.72 & 0.71 & 3.16 & 3.49 & 12.85 \\
\hline & Warping included & 725.9 & 1126.5 & 1165.3 & 1203.8 & 1351.2 \\
\hline & Error (\%) & 1.01 & 0.89 & 0.41 & 0.45 & 0.86 \\
\hline \multirow{5}{*}{$\begin{array}{l}2 a=1 \mathrm{~mm} \\
2 b=0.8 \mathrm{~mm}\end{array}$} & FEM & 888.27 & 1135.3 & 1318.4 & 1369.8 & 1654.8 \\
\hline & Warping ignored & 901.25 & 1133.4 & 1316.8 & 1366.6 & 1677.5 \\
\hline & Error (\%) & 1.46 & 0.16 & 0.12 & 0.23 & 1.40 \\
\hline & Warping included & 881.50 & 1132.6 & 1309.2 & 1358.4 & 1641.9 \\
\hline & Error (\%) & 0.76 & 0.23 & 0.70 & 0.82 & 0.77 \\
\hline \multirow{5}{*}{$\begin{array}{l}2 a=1 \mathrm{~mm} \\
2 b=1 \mathrm{~mm}\end{array}$} & FEM & 1006.7 & 1142.0 & 1396.9 & 1454.8 & 1863.0 \\
\hline & Warping ignored & 996.82 & 1136.7 & 1382.5 & 1437.9 & 1845.2 \\
\hline & Error (\%) & 0.98 & 0.46 & 1.03 & 1.16 & 0.95 \\
\hline & Warping included & 996.66 & 1136.4 & 1382.2 & 1437.8 & 1845.0 \\
\hline & Error (\%) & 0.99 & 0.49 & 1.05 & 1.17 & 0.96 \\
\hline \multirow{5}{*}{$\begin{array}{l}2 a=0.8 \mathrm{~mm} \\
2 b=1 \mathrm{~mm}\end{array}$} & FEM & 886.69 & 913.84 & 1152.0 & 1200.5 & 1618.1 \\
\hline & Warping ignored & 902.91 & 913.55 & 1149.1 & 1196.3 & 1633.7 \\
\hline & Error (\%) & 1.83 & 0.03 & 0.25 & 0.35 & 0.96 \\
\hline & Warping included & 882.74 & 912.38 & 1146.2 & 1192.5 & 1610.8 \\
\hline & Error (\%) & 0.45 & 0.16 & 0.47 & 0.67 & 0.45 \\
\hline \multirow{5}{*}{$\begin{array}{l}2 a=0.6 \mathrm{~mm} \\
2 b=1 \mathrm{~mm}\end{array}$} & FEM & 688.94 & 708.39 & 885.52 & 924.77 & 1279.0 \\
\hline & Warping ignored & 685.18 & 819.72 & 891.44 & 934.60 & 1291.5 \\
\hline & Error (\%) & 0.55 & 15.72 & 0.67 & 1.06 & 0.98 \\
\hline & Warping included & 684.82 & 729.04 & 881.56 & 919.73 & 1279.3 \\
\hline & Error (\%) & 0.59 & 2.92 & 0.45 & 0.55 & 0.02 \\
\hline \multirow{5}{*}{$\begin{array}{l}2 a=0.4 \mathrm{~mm} \\
2 b=1 \mathrm{~mm}\end{array}$} & FEM & 458.34 & 528.46 & 599.48 & 627.81 & 864.93 \\
\hline & Warping ignored & 485.90 & 609.77 & 627.59 & 772.46 & 869.75 \\
\hline & Error (\%) & 6.02 & 15.86 & 4.69 & 23.04 & 0.56 \\
\hline & Warping included & 457.66 & 527.83 & 598.45 & 625.73 & 862.19 \\
\hline & Error (\%) & 0.15 & 0.11 & 0.17 & 0.33 & 0.32 \\
\hline
\end{tabular}




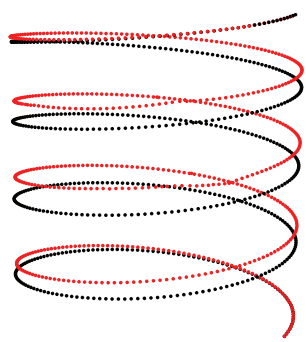

(1)

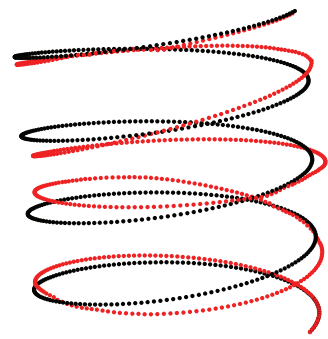

(2)

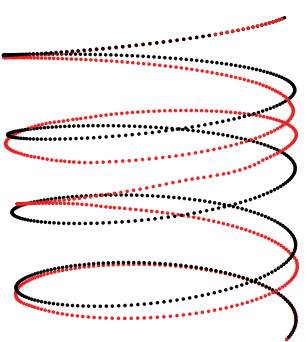

(3)

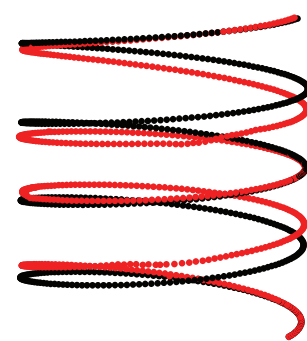

(4)

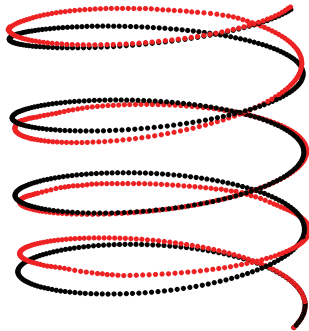

(5)

Figure 4: First five mode shapes of a die spring: (1) first mode (523.25 Hz); (2) second mode (897.71 Hz); (3) third mode (914.36 Hz); (4) fourth mode $(973.88 \mathrm{~Hz})$; (5) fifth mode $(1118.41 \mathrm{~Hz})$.

TABLE 4: Change law of the warping effect on natural frequencies with respect to the diameter of the cylinder.

\begin{tabular}{|c|c|c|c|c|c|c|}
\hline \multicolumn{2}{|c|}{ Mode number $(\mathrm{Hz})$} & 1 & 2 & 3 & 4 & 5 \\
\hline \multirow{5}{*}{$R=4 \mathrm{~mm}$} & FEM & 836.61 & 1419.6 & 1446.5 & 1553.7 & 1760.4 \\
\hline & Warping ignored & 1162.25 & 1574.61 & 1629.74 & 1752.98 & 2089.33 \\
\hline & Error (\%) & 38.92 & 10.92 & 12.67 & 12.83 & 18.68 \\
\hline & Warping included & 818.03 & 1402.37 & 1428.08 & 1522.45 & 1746.51 \\
\hline & Error (\%) & 2.22 & 1.21 & 1.27 & 2.01 & 0.79 \\
\hline \multirow{5}{*}{$R=6 \mathrm{~mm}$} & FEM & 366.39 & 625.73 & 638.26 & 680.81 & 778.55 \\
\hline & Warping ignored & 517.72 & 701.37 & 726.15 & 780.18 & 930.54 \\
\hline & Error (\%) & 41.30 & 12.09 & 13.77 & 14.60 & 19.51 \\
\hline & Warping included & 363.61 & 623.34 & 635.98 & 676.02 & 777.59 \\
\hline & Error (\%) & 0.76 & 0.38 & 0.36 & 0.70 & 0.12 \\
\hline \multirow{5}{*}{$R=8 \mathrm{~mm}$} & FEM & 205.48 & 351.70 & 358.68 & 382.02 & 438.26 \\
\hline & Warping ignored & 291.77 & 394.48 & 408.34 & 439.17 & 523.85 \\
\hline & Error (\%) & 41.99 & 12.16 & 13.85 & 14.96 & 21.58 \\
\hline & Warping included & 204.59 & 350.92 & 357.63 & 380.31 & 437.40 \\
\hline & Error (\%) & 0.43 & 0.22 & 0.29 & 0.45 & 0.20 \\
\hline \multirow{5}{*}{$R=10 \mathrm{~mm}$} & FEM & 131.12 & 224.78 & 229.20 & 243.94 & 280.38 \\
\hline & Warping ignored & 186.48 & 252.72 & 261.06 & 281.29 & 335.25 \\
\hline & Error (\%) & 42.22 & 12.43 & 13.90 & 15.31 & 19.57 \\
\hline & Warping included & 130.43 & 224.46 & 228.59 & 243.07 & 280.32 \\
\hline & Error (\%) & 0.53 & 0.14 & 0.27 & 0.36 & 0.02 \\
\hline
\end{tabular}

and $b$ in the $\eta$-direction. The warping function of SaintVenant's torsion of the rectangular cross section is

$$
\begin{aligned}
& \varphi(\xi, \eta)=\xi \eta-\frac{32 a^{2}}{\pi^{3}} \\
& \quad \cdot \sum_{n=0}^{\infty} \frac{(-1)^{n} \sinh ((2 n+1) \pi \eta / 2 a)}{(2 n+1)^{3} \cosh ((2 n+1) \pi b / 2 a)} \sin \frac{(2 n+1) \pi \xi}{2 a} .
\end{aligned}
$$

Utilizing ANSYS program in this analysis, the die spring is partitioned into 720 solid elements (the type is solid 45 in ANSYS); the total number of node is 1452 . Let $R=5 \mathrm{~mm}$, $\bar{\alpha}=5^{\circ}, n=4,2 a=1 \mathrm{~mm}$, and $2 b=0.4 \mathrm{~mm}$. The first five mode shapes and normal mode shapes of the generalized warping coordinate of this spring are shown in Figures 4 and 5 , respectively. 
TABLE 5: Change law of the warping effect on natural frequencies with respect to the number of coils.

\begin{tabular}{|c|c|c|c|c|c|c|}
\hline \multicolumn{2}{|c|}{ Mode number $(\mathrm{Hz})$} & 1 & 2 & 3 & 4 & 5 \\
\hline \multirow{5}{*}{$n=2$} & FEM & 984.59 & 1400.9 & 1738.5 & 2168.0 & 2259.2 \\
\hline & Warping ignored & 1332.77 & 1713.92 & 1848.66 & 2286.35 & 2336.40 \\
\hline & Error (\%) & 35.36 & 22.34 & 6.34 & 5.46 & 3.42 \\
\hline & Warping included & 973.17 & 1406.73 & 1746.22 & 2263.04 & 2271.58 \\
\hline & Error (\%) & 1.16 & 0.42 & 0.44 & 4.38 & 0.55 \\
\hline \multirow{5}{*}{$n=4$} & FEM & 521.34 & 899.54 & 915.97 & 970.21 & 1129.5 \\
\hline & Warping ignored & 745.84 & 1009.30 & 1044.17 & 1122.22 & 1338.65 \\
\hline & Error (\%) & 43.06 & 12.20 & 13.99 & 15.67 & 18.52 \\
\hline & Warping included & 523.25 & 897.71 & 914.36 & 973.88 & 1118.41 \\
\hline & Error (\%) & 0.37 & 0.20 & 0.17 & 0.38 & 0.98 \\
\hline \multirow{5}{*}{$n=6$} & FEM & 359.89 & 521.12 & 529.55 & 697.79 & 771.62 \\
\hline & Warping ignored & 504.25 & 574.71 & 588.36 & 765.78 & 963.49 \\
\hline & Error (\%) & 40.11 & 10.28 & 11.10 & 9.76 & 24.87 \\
\hline & Warping included & 353.34 & 515.50 & 523.97 & 685.21 & 764.24 \\
\hline & Error (\%) & 1.82 & 1.08 & 1.05 & 1.80 & 0.96 \\
\hline \multirow{5}{*}{$n=8$} & FEM & 270.56 & 330.04 & 335.05 & 533.20 & 582.48 \\
\hline & Warping ignored & 359.73 & 364.84 & 393.39 & 577.06 & 731.52 \\
\hline & Error (\%) & 32.96 & 10.54 & 17.41 & 8.23 & 25.59 \\
\hline & Warping included & 265.39 & 326.26 & 331.55 & 523.17 & 764.24 \\
\hline & Error (\%) & 1.91 & 1.14 & 1.04 & 1.88 & 0.86 \\
\hline
\end{tabular}

TABLE 6: Change law of the warping effect on natural frequencies with respect to the helix pitch angle.

\begin{tabular}{|c|c|c|c|c|c|c|}
\hline \multicolumn{2}{|c|}{ Mode number $(\mathrm{Hz})$} & \multirow{2}{*}{$\frac{1}{533.77}$} & \multirow{2}{*}{$\frac{2}{966.74}$} & \multirow{2}{*}{$\frac{3}{980.62}$} & \multirow{2}{*}{$\begin{array}{c}4 \\
993.31\end{array}$} & \multirow{2}{*}{$\frac{5}{1120.5}$} \\
\hline \multirow{5}{*}{$\bar{\alpha}=4^{\circ}$} & FEM & & & & & \\
\hline & Warping ignored & 748.56 & 1082.97 & 1099.23 & 1137.02 & 1345.44 \\
\hline & Error (\%) & 40.24 & 12.02 & 12.09 & 14.47 & 20.07 \\
\hline & Warping included & 525.88 & 957.13 & 971.50 & 978.46 & 1112.27 \\
\hline & Error (\%) & 1.50 & 0.99 & 0.93 & 1.49 & 0.73 \\
\hline \multirow{5}{*}{$\bar{\alpha}=6^{\circ}$} & FEM & 528.43 & 840.48 & 862.36 & 981.13 & 1125.3 \\
\hline & Warping ignored & 740.62 & 936.78 & 973.11 & 1122.27 & 1330.55 \\
\hline & Error (\%) & 40.15 & 11.46 & 12.84 & 14.39 & 18.24 \\
\hline & Warping included & 521.71 & 834.46 & 855.53 & 970.49 & 1119.82 \\
\hline & Error (\%) & 1.27 & 0.72 & 0.79 & 1.08 & 0.49 \\
\hline \multirow{5}{*}{$\bar{\alpha}=8^{\circ}$} & FEM & 520.35 & 722.19 & 751.51 & 966.25 & 1118.0 \\
\hline & Warping ignored & 722.42 & 809.66 & 856.58 & 1119.07 & 1307.31 \\
\hline & Error (\%) & 38.83 & 12.11 & 13.95 & 15.82 & 16.93 \\
\hline & Warping included & 515.27 & 718.51 & 747.84 & 957.19 & 1115.40 \\
\hline & Error (\%) & 0.97 & 0.51 & 0.51 & 0.94 & 0.23 \\
\hline \multirow{5}{*}{$\bar{\alpha}=10^{\circ}$} & FEM & 509.13 & 625.76 & 663.87 & 949.78 & 1106.1 \\
\hline & Warping ignored & 676.70 & 703.33 & 787.78 & 1113.24 & 1273.52 \\
\hline & Error (\%) & 32.91 & 12.40 & 18.66 & 17.21 & 15.14 \\
\hline & Warping included & 504.25 & 622.41 & 659.50 & 941.67 & 1102.38 \\
\hline & Error (\%) & 0.96 & 0.54 & 0.66 & 0.85 & 0.34 \\
\hline
\end{tabular}




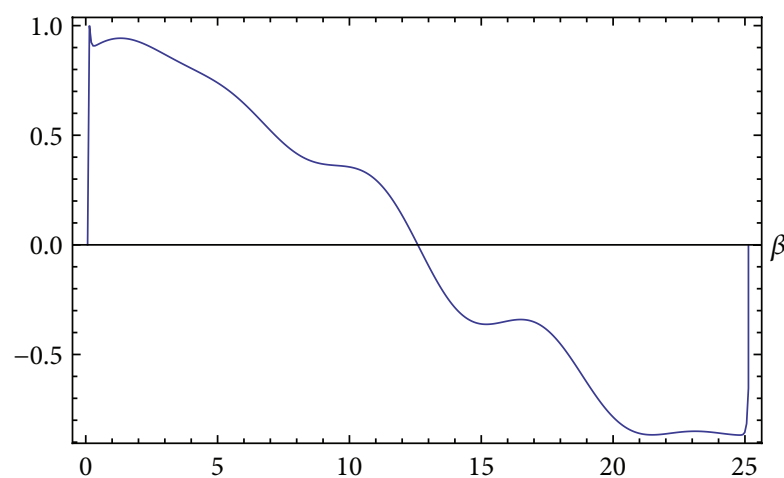

$(1)$

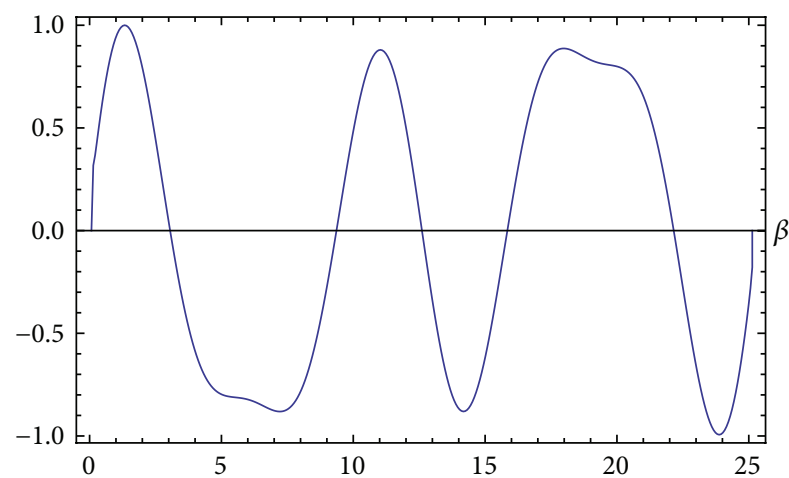

(3)

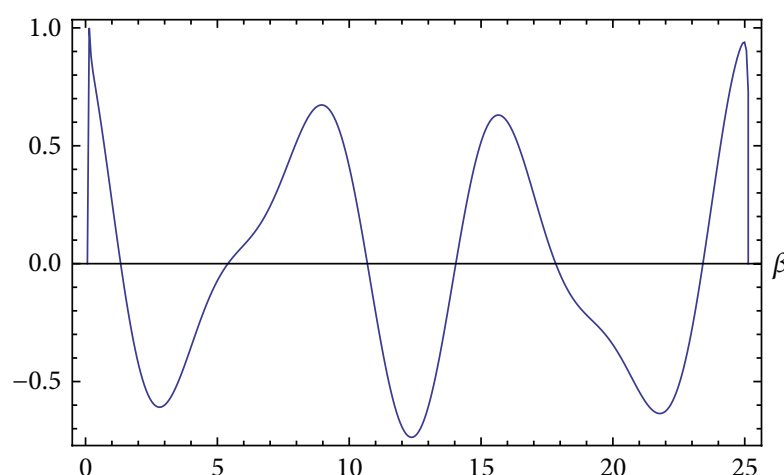

(2)

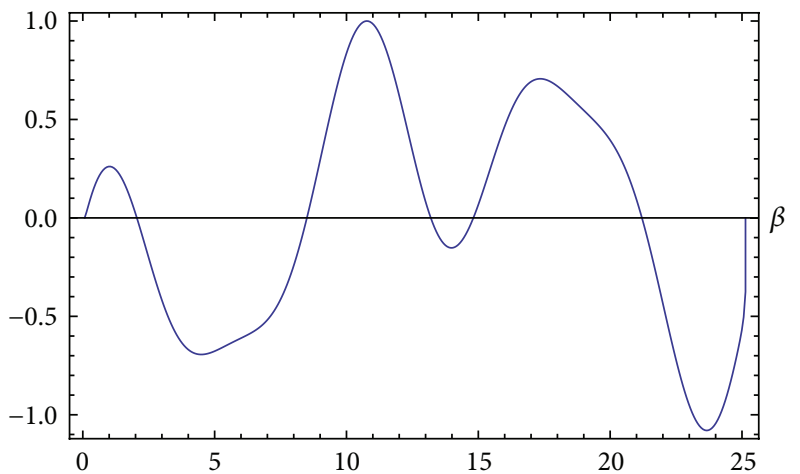

$(4)$

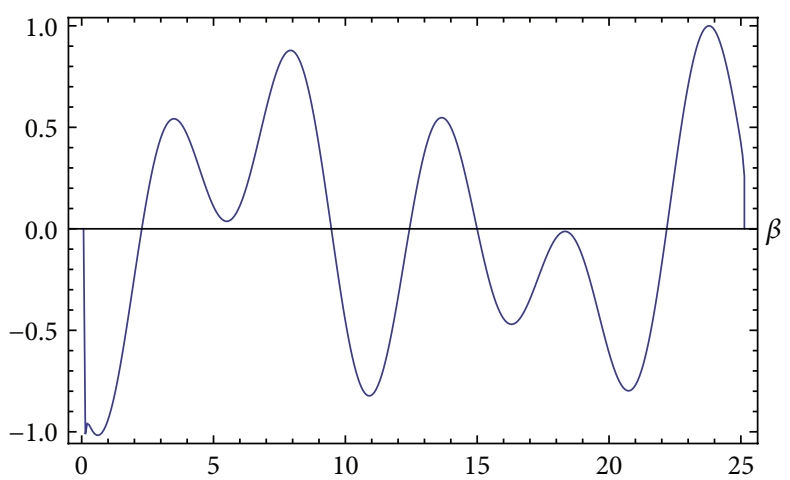

(5)

FiguRE 5: First five normal mode shapes of the generalized warping coordinate of a die spring: (1) first mode (523.25 Hz); (2) second mode $(897.71 \mathrm{~Hz})$; (3) third mode $(914.36 \mathrm{~Hz})$; (4) fourth mode $(973.88 \mathrm{~Hz})$; (5) fifth mode $(1118.41 \mathrm{~Hz})$.

Table 2 shows that the effect of warping deformations on the natural frequencies of the spring is very significant. Therefore, the errors can be unacceptably large when the warping effect is neglected. The necessity to account for the warping effect in the free vibration analysis of this spring is clearly evident.

Example 3. Utilizing ANSYS code in the analysis, the die springs of this example are partitioned into 720 solid 45 elements; the total number of node is 1452 . Let $R=5 \mathrm{~mm}$, $\bar{\alpha}=5^{\circ}$, and $n=4$.
From Table 3, we find the following phenomena:

(1) The natural frequencies of the spring increase with increase of the cross-sectional areas.

(2) If spring's cross-sectional areas keep fixed, the different arrangements of the cross section will have significant effect on the natural frequencies. When the cross section is narrower, the differences between the natural frequencies are greater.

(3) It is suggested to consider the warping deformation in die spring's dynamic analysis when the aspect ratio of 
the rectangular cross section is $a: b=1: 0.6$. When the aspect ratio is $a: b=1: 0.4$, the influence of warping must be considered.

Example 4. Consider $\bar{\alpha}=5^{\circ}, n=4,2 a=1 \mathrm{~mm}$, and $2 b=$ $0.4 \mathrm{~mm}$.

Example 5. Consider $R=5 \mathrm{~mm}, \bar{\alpha}=5^{\circ}, 2 a=1 \mathrm{~mm}$, and $2 b=0.4 \mathrm{~mm}$.

Example 6. Consider $R=5 \mathrm{~mm}, n=4,2 a=1 \mathrm{~mm}$, and $2 b=0.4 \mathrm{~mm}$.

Tables $4-6$ show that when $\bar{\alpha}, n$, and $R / 2 a$ increase, the spring length increases and the frequencies decrease. However, the variety rule of warping effect on these factors is not obvious.

\section{Conclusion}

This study presents the change laws of warping effect on natural frequencies with respect to spring parameters. The warping effect exhibits the most remarkable changes with the variation of the height-to-width ratio of the cross section. However, this effect is not fairly sensitive to the changes in other parameters, and it is particularly significant when the cross section is relatively narrow regardless of changes in other parameters. In this case, approximately $40 \%$ of errors may occur if warping is neglected.

\section{Competing Interests}

The authors declare that they have no competing interests.

\section{Acknowledgments}

This work is partially supported by National Natural Science Foundation of China (11402090 and 51209094) and the Science and Technology Planning Project of Zhengzhou City of China (no. 153PKJGG111).

\section{References}

[1] D. Fakhreddine, T. Mohamed, A. Said, D. Abderrazek, and H. Mohamed, "Finite element method for the stress analysis of isotropic cylindrical helical spring," European Journal of Mechanics-A/Solids, vol. 24, no. 6, pp. 1068-1078, 2005.

[2] V. Yildirin, "Investigation of parameters affecting free vibration frequency of helical springs," International Journal for Numerical Methods in Engineering, vol. 39, no. 1, pp. 99-114, 1996.

[3] J. Lee, "Free vibration analysis of cylindrical helical springs by the pseudospectral method," Journal of Sound and Vibration, vol. 302, no. 1-2, pp. 185-196, 2007.

[4] X.-G. Wu, B.-L. Zheng, P.-F. He, and S.-G. Liu, "Equilibrium equations for 3D critical buckling of helical springs," Applied Mathematics and Mechanics, vol. 33, no. 8, pp. 1049-1058, 2012.

[5] I. Ecsedi, "Some analytical solutions for Saint-Venant torsion of non-homogeneous cylindrical bars," European Journal of Mechanics A: Solids, vol. 28, no. 5, pp. 985-990, 2009.
[6] L. E. Becker and W. L. Cleghorn, "Buckling behavior of rectangular-bar helical compression springs," Journal of Applied Mechanics, vol. 61, no. 2, pp. 491-493, 1994.

[7] W. Busool and M. Eisenberger, "Free vibration of helicoidal beams of arbitrary shape and variable cross section," Journal of Vibration and Acoustics, vol. 124, no. 3, pp. 397-409, 2002.

[8] K. Girgin, "Free vibration analysis of non-cylindrical helices with, variable cross-section by using mixed FEM," Journal of Sound and Vibration, vol. 297, no. 3-5, pp. 931-945, 2006.

[9] V. Yildirim, "An efficient numerical method for predicting the natural frequencies of cylindrical helical springs," International Journal of Mechanical Sciences, vol. 41, no. 8, pp. 919-939, 1999.

[10] M. Taktak, F. Dammak, S. Abid, and M. Haddar, "A finite element for dynamic analysis of a cylindrical isotropic helical spring," Journal of Mechanics of Materials and Structures, vol. 3, no. 4, pp. 641-658, 2008.

[11] A. M. Yu and Y. Hao, "Free vibration analysis of cylindrical helical springs with noncircular cross-sections," Journal of Sound and Vibration, vol. 330, no. 11, pp. 2628-2639, 2011.

[12] A. M. Yu and Zh. H. Qu, "The study of variational principle in naturally curved and twisted slender beams," Journal of Shanghai Technical College of Metallurgy, vol. 15, no. 3, pp. 1-8, 1994 (Chinese).

[13] A. M. Yu, C. J. Yang, and G. H. Nie, "Analytical formulation and evaluation for free vibration of naturally curved and twisted beams," Journal of Sound and Vibration, vol. 329, no. 9, pp. 13761389, 2010.

[14] V. Yildirim, "Free vibration analysis of non-cylindrical coil springs by combined use of the transfer matrix and the complementary functions methods," Communications in Numerical Methods in Engineering, vol. 13, no. 6, pp. 487-494, 1997.

[15] B. G. Liu, Perturbation RICCATI Transfer Matrix Method for One Dimensional Structure with Parameter Uncertainties and Its Applications, Chongqing University, Chongqing, China, 2002 (Chinese).

[16] A. M. Yu and Y. Hao, "Effect of warping on natural frequencies of symmetrical cross-ply laminated composite non-cylindrical helical springs," International Journal of Mechanical Sciences, vol. 74, pp. 65-72, 2013. 


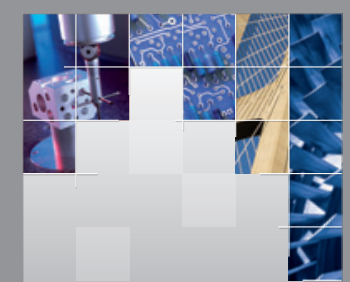

\section{Enfincering}
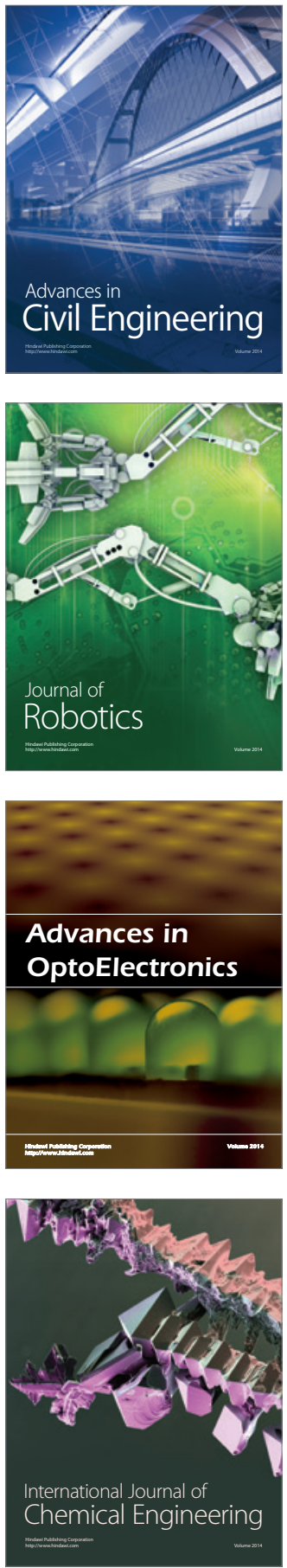

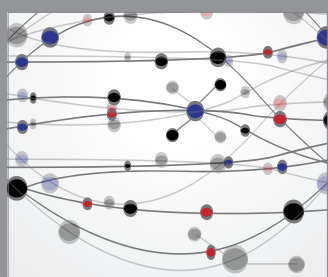

The Scientific World Journal

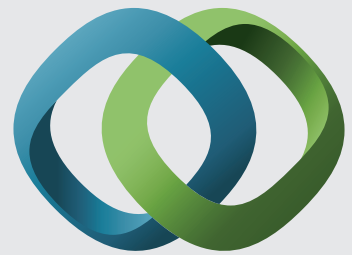

\section{Hindawi}

Submit your manuscripts at

http://www.hindawi.com
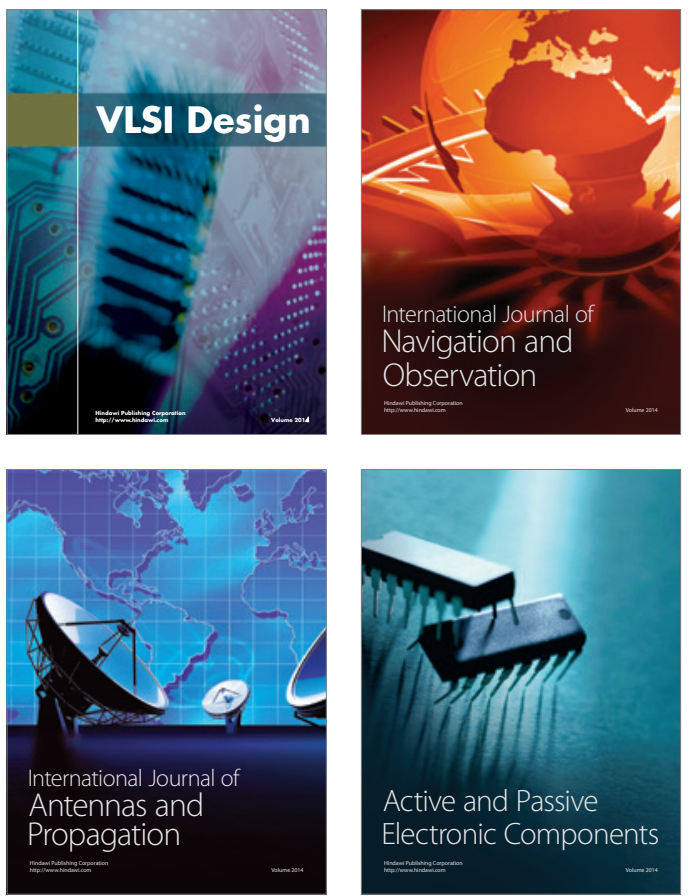
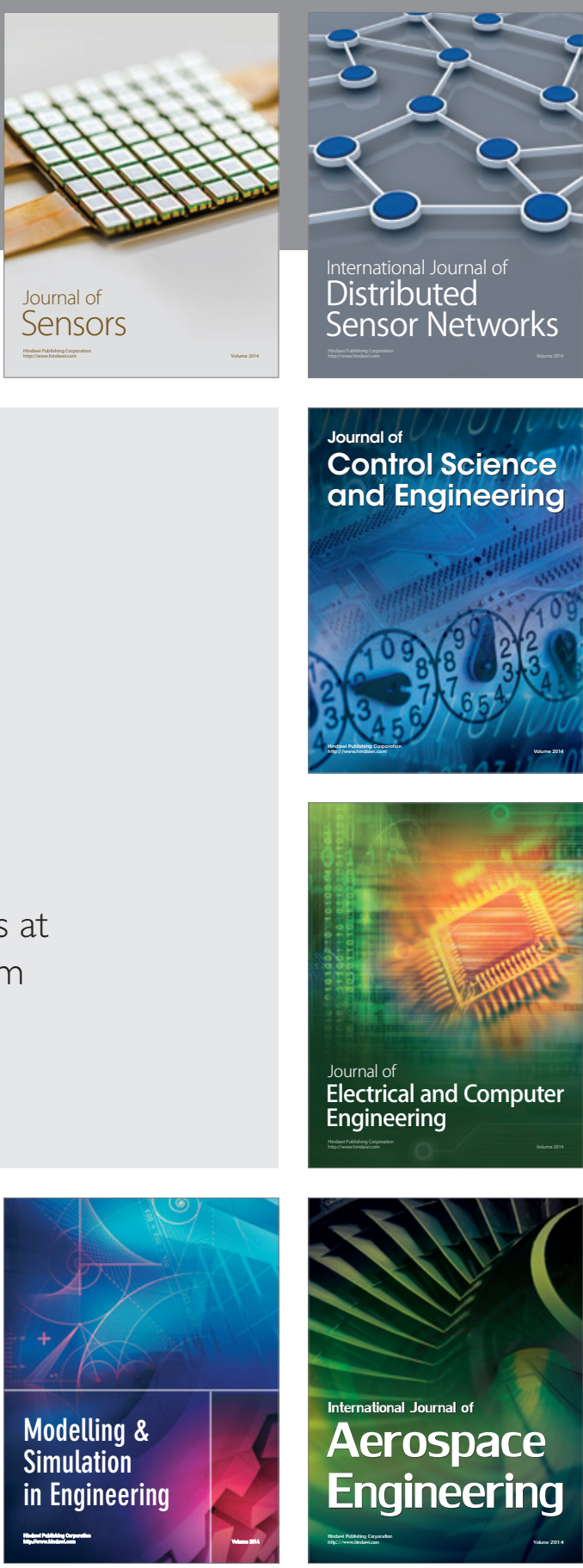

International Journal of

Distributed

Sensor Networks

Journal of

Control Science

and Engineering
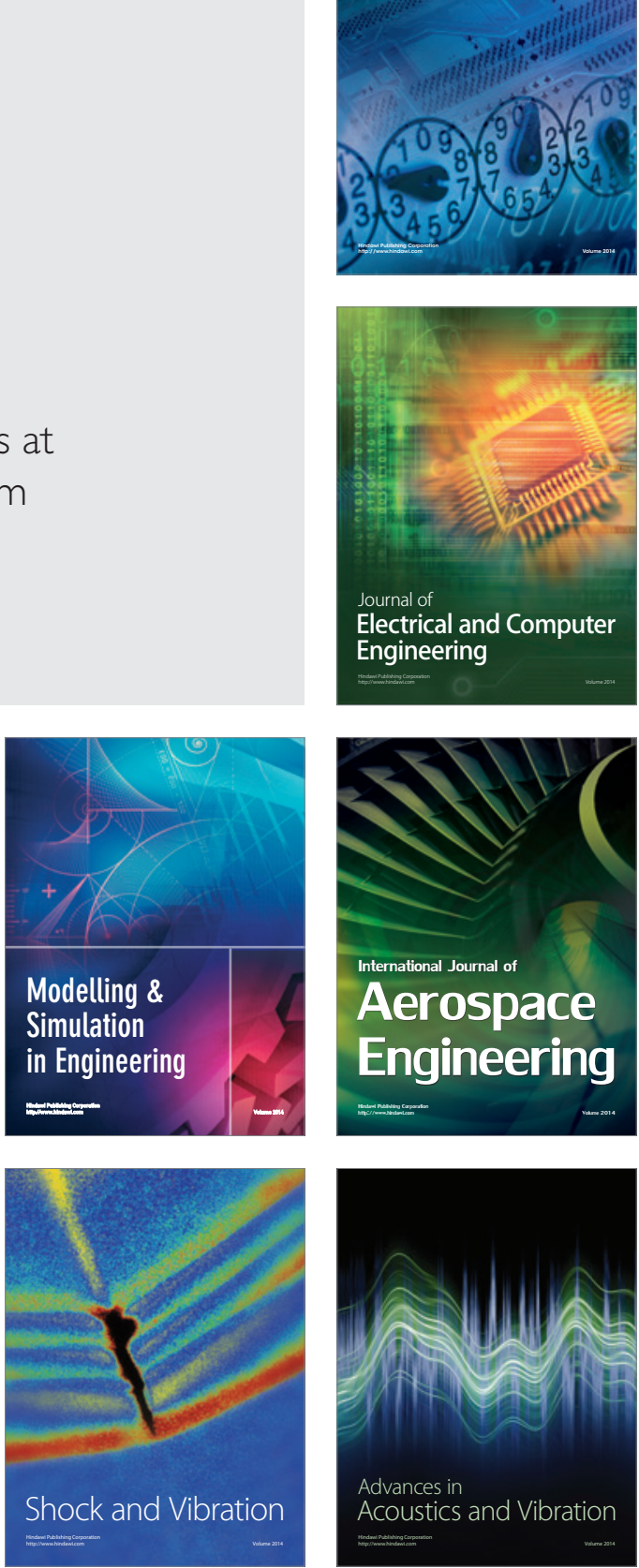